\title{
Marion Kloep, Leo B. Hendry, Rachel Taylor, and Ian Stuart- Hamilton: Development From Adolescence to Early Adulthood: A Dynamic Systemic Approach to Transitions and Transformations
}

\author{
Psychology Press, Hove, East Sussex, 2016, 164 pp, ISBN-13: 978-0415640091
}

\author{
Emilia Rodriguez ${ }^{1}$
}

Received: 12 October 2016/Accepted: 13 October 2016/Published online: 21 November 2016

(C) Springer International Publishing 2016

Development from Adolescence to Early Adulthood uses examples of concepts and explanations to argue that the most effective way of addressing human changes, specifically the changes involved in adolescent development, is through a dynamic systems approach. Human development has traditionally been studied using a model based on stages, single variables, and averages. While the dynamic systems approach is relatively new and steers away from the research methods that have been used in the past, Kloep et al. are confident of the idea that this modern approach is significantly more accurate than traditional methods. The authors advocate for this method not only for its improved appropriateness in providing a better understanding of data, but also because it allows multiple fields in the social sciences to collaborate. The book intends to persuade social science researchers to adapt this entirely innovative way of looking at stages of development and social contexts by focusing on the individual and their specific context.

In chapter one, called "Looking at Transitions", the authors introduce the dynamic systems approach by describing the work of prominent researchers who had similar views, presenting opposing views, and providing concrete examples that support it as a more effective approach over the current method. The chapter begins with an explanation of development in terms of cellular differentiation, which promotes the idea that human beings belong to a system that is constantly changing, and that variation is what drives these changes. The authors argue that behaviors should not be deemed good or bad because there is a context to every situation and all behaviors have

Emilia Rodriguez

emirodri@indiana.edu

Indiana University Bloomington, Bloomington, IN, USA the capacity to be beneficial or dangerous to the individual depending on the context. This exhibits how studies focusing on one variable usually fail to offer accurate results because variance is often recorded as error instead of as a finding. Furthermore, the results tend to leave out contextual information that would be helpful in explaining the variance obtained. The authors continue by further describing the weaknesses of current methods for studying development and the benefits to dynamic systems. The terms used in the dynamic systems approach such as "attractor points", "transition states", and "cascading constraints" are defined to present the reader with tools to understand subsequent chapters. The authors emphasize that, unlike previous methods, dynamic systems determine transition phases not by age, but by culture, gender, social class, and genetics. Lastly, the authors discuss how data from these studies can be empirically measured without using aggregate methods that solely produce mean values of the data set. The authors are convinced that this approach will eventually be widely accepted in the future. However, it is difficult to persuade psychologists who are set in their ways.

Chapter two, named "Social contexts of development", displays the overlapping relationship between social psychology, developmental psychology, and dynamic systems. This chapter functions to demonstrate how the dynamic systems approach would improve the results of already researched topics and will be useful for future studies. This demonstration uses social concepts to show that social psychology concepts can be applied to dynamic systems, which would help psychologists describe their variations. It also argues that developmental and social psychology would benefit from adapting the dynamic systems approach. These three fields together can potentially provide the best explanation for adolescent development 
because of the incorporation of multiple perspectives. The chapter begins its discussion with a description of the three stages of social psychology and explains that these levels are generally inter-related. Next, the authors define an "attractor point" with an example regarding group identification. The connection between social psychology and dynamic systems is presented with the existence of attractor points and constraining cascades in explaining attitudes and behavior. Another component of dynamic systems that is seen in social psychology is the idea of discontinuous change which is illustrated with an example of group identification in music fan groups. Later, the chapter addresses the problem social psychology has in incorporating social aspects and how this problem could be solved. The authors emphasize that measuring attractor points and cascading constraints would elevate the social aspect of social psychology. The following section addresses the idea that developmental and social psychologies are highly related, and collaboration of the two fields would improve the discoveries of common concepts. This idea is demonstrated with two examples of studies using dynamic approaches to social behavior.

Chapter three, entitled “Adolescents' social engagements", uses individual complexity involved in social challenges of adolescents to provide an example of a subject that would benefit from a dynamic systems approach. The first social concept described is friendship, and the authors emphasize that these relationships deliver several beneficial functions to adolescents which are influenced by various external factors. The chapter also presents research by Veenstra et al. (2010), which shows that groups promoting risky behavior can actually benefit the adolescent by providing strong social ties. These ties create a sense of belonging and enhance feelings of selfworth among other attributes. The next section describes how group-identification contributes to self-identification. Here the authors detail other works to show the complexity of social identity, especially in adolescents. This complexity is detailed further with an explanation for why multiple identities in adolescence promotes a developed social identity. The chapter then addresses the influences of social media in adolescent friendships and how online and offline entities interact. Finally, the chapter concludes with a discussion on adolescents and romantic relationships. The researches in these areas are related to context and help to advocate for the dynamic systems approach.

In chapter four, called "Compliments, lies and other social skills", the authors discuss another area of adolescent development: the influence of deception in social development. The authors provide a positive perspective on deception throughout the chapter beginning with disclosure and secrecy. Research by Grice et al. (1975) shows that nondisclosure is usually seen as a negative behavior, but too much disclosure tends to effect the adolescent's social skills and causes embarrassment. In addition, classifications of information domains as moral, social-conventional, and personal, can control the frequency of disclosure. These classifications also function to explain emotional rigidity in adolescent conflicts. The importance of identity resurfaces in this chapter to explore how offline and online domains aid in identity management. Offline identity management has lacked in research, but have more studies regarding groups. Within-group studies show that impression management is important for social skill development. Online identity management is much richer in research, however, research on online networking as a way to self-present focuses on how there are concerns that should be addressed by parents and teachers. The last section of the chapter directs the reader's attention towards excuses, compliments, and praise in relation to adolescent social skills.

Chapter five, named "Health in adolescence: A lifespan perspective", reinforces the first chapter by revisiting the idea that life development should not be studied in stages, but in change over time. The body starts to decay during adolescence, which is an ongoing process that continues until death. The first section uses tables that demonstrate how income impacts the types of diseases that cause death, and at what age death occurs. Then the authors visit the theme of environment, genetics, and life expectancy. This section expresses how evolutionary pressures has led to adolescent changes (i.e., puberty) to cause characteristics that are not ideal for longer life expectancies that are seen today. Puberty is discussed with the impacts that it has on adolescents. Specifically, females that start puberty earlier than others in their culture have a higher risk of substance abuse and depression. Puberty also increases the likelihood that adolescents will have their sexual debut. The health issues that surround sex include HIV/AIDs and STIs which are more common in adolescents. This could mainly be due to the fact that more people have sex in that age group than other age groups. The authors then proceed to talk about tobacco use where the frequency has to do with cultural norms. Substance abuse, however, has a different trend. This behavior is commonly started in adolescence, but does not necessarily mean that it will continue in the individual's adult life. The following section addresses how adolescence is a time period when many learning disability disorders are recognized as well as mental health issues. For both of these areas of health, it is crucial to identify signs early on to allow proper treatment and an improvement in the individual's quality of life. Adolescent health in every sector is dependent on dynamic systems. In other words, there are many influences to the manifestations of diseases and behaviors surrounding health.

In chapter six, titled "Transitions to the world of work", Kloep et al. highlight the path to adulthood through the 
workforce using dynamic systems. The chapter starts with a study by Vondracek et al. (2014) that says innate features of an individual inter-connect to produce a certain skill set that guides their career path. Social factors also influence an adolescent's transition to work. These factors include parents, romantic relationships, neighborhood, local community, and level of masculinity/femininity. The next section addresses the amount of educational training involved in preparing students for job-related skills. Then, the authors emphasize how technology experience and welfare systems benefit adolescents who are trying to transition to work. Accordingly, young people from disadvantaged backgrounds usually fail to achieve a higher level work trajectory. Furthermore, many employers are struggling to find workers with the necessary skills to do their jobs properly. In addition, entry-level jobs are being replaced by machines that are able to do the same jobs as humans. Consequently, unemployment causes individual and complex results that can be explored with a more dynamic approach. The authors conclude that the transition into adulthood relies on individual backgrounds which influences the adolescent's ability and motivation for pursuing a future.

Chapter seven, "Family dynamics and transitions towards adult independence", is the final illustration for the use of dynamic systems. This chapter moves on from work to the area of familial relationships. The beginning section of this chapter emphasizes the need to highlight each member of the family's experience. Their shared environment is still diverse in that all family members are interacting with one another and forming specific roles. Families are constantly adapting as changes occur like economic crises, puberty, menopause, mid-life crises, and divorce. The process of an adolescent's separation from home is discussed along with the repercussions that are associated with different levels of independence. The next section touches on romantic relationships, which is usually the next step in a young adult's life after separation from families. Young people start to utilize distinct strategies for dating and explore their sexuality and different commitment styles. The authors note that research is scant in regards to adolescent romantic relationships because they are not taken seriously. However, these patterns set the stage for romantic pursuits later in life. Dating violence in particular is a serious issue that adolescents face with respect to romantic relationships, and this area would profit from more research attention. Babies cause a novel type of stress for the new parents, which are accompanied by adaptations in their original daily routine. This stress is intensified when the baby is unplanned, which can have serious negative effects on the parents depending on their context.
Lastly, chapter eight, entitled "Dynamic systems theory: Changing the paradigm?" summarizes the previous chapters by explaining what this novel approach contributes to the field of social science research. It starts with a statement about how the minimal research that there is has created new results with existing adolescent studies. One motive for why the field needs a new way of approaching research is to move away from the theory of stages that makes adolescents seem entirely separate from adults. Brain research studies have produced many fallacies that reinforce the results of stage theory research in adolescents. The authors revert back to previous arguments about defining behavior as good or bad. They attempt to persuade the audience by stating that actions should be regarded as adaptive in relation to the individual's context. The authors then propose that development could be seen as a result of a dissatisfaction instead of a conflict which reflects Maslow's (1943) work. The following section communicates an individual-based method for creating interventions that can support adolescents academically. The final section explains that new terminology is needed to provide universal terms for all related fields that could adapt this approach.

Each chapter of this book contributes evidence for why dynamic systems are a more adequate way to approach developmental changes in adolescents. In addition, the authors advocate for a more positive view of adolescent behaviors that are normally seen as negative. The sections in each chapter highlight social research examples that adopt a contextual structure. Although most social scientists may well be difficult to persuade, this book provides excellent examples and reasoning for why the current research methods are unsuccessful. In addition, the book provides methods for applying dynamic approaches to these examples. Despite these strengths, the authors made use of several different areas of research when readers would have benefitted from a deeper focus on less areas to present the data and use of dynamic systems more clearly. Overall, however, the book has an eye-opening perspective that hopefully will make social science researchers think twice before continuing to use their traditional methods.

\section{Compliance with Ethical Standards}

Conflict of interest The author reports none.

\section{References}

Grice, H. P., Cole, P., \& Morgan, J. L. (1975). Syntax and semantics. Logic and Conversation, 3, 41-58.

Maslow, A. H. (1943). A theory of human motivation. Psychological Review, 50(4), 370-396.

Veenstra, R., Huitsing, G., Dijkstra, J. K., \& Lindenberg, S. (2010). Friday on my mind: The relation of partying with antisocial 
behavior of early adolescents. The TRAILS Study. Journal of Research on Adolescence, 20(2), 420-431.

Vondracek, F. W., Ford, D. H., \& Porfeli, E. J. (2014). A living systems theory of vocational behavior and development. In F.
W. Vondracek, D. H. Ford, \& E. J. Porfeli (Eds.), A living systems theory of vocational behavior and development (pp. 55-80). Boston: Sense Publishers. 